\title{
Eco-friendly water-based $\lambda$-cyhalothrin polydopamine microcapsule suspension with high adhesion on foliage for reducing pesticides loss
}

Tong Wü ${ }^{\sharp a}$, Xialun Fang ${ }^{\sharp b}$, Ying Yang ${ }^{\mathrm{b}}$, Wenyan Meng ${ }^{\mathrm{b}}$, Pengji Yao ${ }^{\mathrm{b}}$, Qin Liu ${ }^{\mathrm{c}}$, Bo Zhang ${ }^{\mathrm{d}}$, Feng Liu ${ }^{\mathrm{e}}$, Aihua Zou*b, Jiagao Cheng*a

a Shanghai Key Laboratory of Chemical Biology, School of Pharmacy, East China University of Science and Technology, Shanghai 200237, China

${ }^{\mathrm{b}}$ Shanghai Key Laboratory of Functional Materials Chemistry, State Key Laboratory of Bioreactor Engineering and Molecular Engineering, East China University of Science and Technology, Shanghai 200237, China

c State Key Laboratory of Bioreactor Engineering, East China University of Science and Technology, Shanghai, 200237, China

d The Key Laboratory of Resource Chemistry of Ministry of Education, Shanghai Engineering Research Center of Green Energy Chemical Engineering, College of Chemistry and Materials Science, Shanghai Normal University, Shanghai, 200234, P. R. China.

e Shandong Provincial Key Laboratory for Biology of Vegetable Diseases and Insect Pests, College of Plant Protection, Shandong Agricultural University, Tai'an, 271001, China

\section{${ }^{*}$ Corresponding author}

Aihua Zou, Jiagao Cheng

East China University of Science and Technology

Shanghai, 200237, China

E-mail: aihuazou@ecust.edu.cn; jgcheng@ecust.edu.cn

Tel/Fax: +86-21-64252231; +86-21-64251348 


\section{Preparation of $\mathbf{2 . 5 \%} \lambda$-cyhalothrin (LC)-polydopamine}

\section{(PDA) microcapsule suspension (CS)}

The $2.5 \%$ LC-PDA CS was prepared using LC-PDA microcapsules (MCs) that were previously prepared as the main raw material. ${ }^{l}$ First, $25.0 \%$ dispersant (SP-SC3260) aqueous solution was obtained by mixing $25 \mathrm{~g}$ of SP-SC3260 and $75 \mathrm{~g}$ of water until the system is homogeneous, $1.0 \%$ thickener (xanthan gum) aqueous solution and 1\% antifoam (WACKER SRECN type) aqueous solution were obtained by the same preparation method as mentioned above.

Second, $0.5 \mathrm{~g}$ the LC-PDA MCs, $2.0 \mathrm{~g}$ dispersant (25.0\% SP-SC3260 aqueous solution), $2.0 \mathrm{~g}$ antifoam (1.0\% WACKER SRECN aqueous solution) and $2.0 \mathrm{~g}$ redistilled water were mixed together under constant stirring at $200 \mathrm{rpm}$ with a magnetic stirrer for 1 hour at room temperature. Then, $3.0 \mathrm{~g}$ thickener $(1.0 \%$ xanthan gum aqueous solution), $0.4 \mathrm{~g}$ antifreeze (ethylene glycol) and $0.1 \mathrm{~g}$ preservative (sodium benzoate) was added to the above mixture, and magnetic stirring was carried out at room temperature for 30 minutes to obtain $2.5 \%$ LC-PDA CS.

\section{Method}

\subsection{Screening Method}

\subsubsection{Optimization of the amount of additives by an orthogonal test}

Through screening of a flow point method, $25.0 \%$ wet dispersant aqueous solution (SP-SC3260) and 1.0\% xanthan gum aqueous solution were respectively found to be a suitable wet-dispersing agent and a thickener. Under the conditions of maintaining the microcapsule content $(5.0 \%)$, antifreeze content $(4.0 \%)$, antifoaming 
agent content $(0.2 \%)$ and preservative content $(0.1 \%)$, the proportion of wetting dispersant was changed between $2.5 \%$ and $10.0 \%$, and the proportion of thickener was between $0.1 \%$ and $0.4 \%$. The experimental design scheme was as shown in Table S1.

To screen for the best formula, the prepared samples were first observed at room temperature. Then we further assessed these formulations according to their suspension rate, dumping performance and storage stability.

\subsubsection{Suspension rate measurement}

The suspension rate measurement was according to the national standards about determination method of suspensibility for pesticides. ${ }^{2}$ The LC-PDA CS (1.0 g) was weighed into a beaker containing $50 \mathrm{~mL}$ of standard hard water, and slowly shaken by hand and uniformly moved in a circular motion for 2 minutes. The above solution was poured into a measuring cylinder, covering with a stopper, and kept in a constant temperature water bath for 4 minutes, then diluted with standard hard water to the scale line of the measuring cylinder. With the bottom of the measuring cylinder as the oscillating axis, the measuring cylinder was inverted upside down 30 times in $1 \mathrm{~min}$. After slowly opening the stopper, the measuring cylinder was placed in a water bath $\left(25^{\circ} \mathrm{C}\right)$ for $30 \mathrm{~min}$. Next, the $9 / 10$ suspension in the graduated cylinder was removed with a pipette within $10-15 \mathrm{~s}$, the solid was separated by filtration, and dried. Then, the weight of the dried microcapsule suspension in the $1 / 10$ suspension at the bottom of the measuring cylinder was $\mathrm{w}_{1}(\mathrm{~g})$. All measurements were performed in triplicate. The suspension rate was calculated using formula (1). 


$$
\text { Suspension rate }(\%)=\left(\mathrm{w}-\mathrm{w}_{1}\right) / \mathrm{w} \times 1.11 \times 100 \%
$$

Where $\mathrm{w}$ was the mass of the sample weighed by the suspensions.

\subsubsection{Dumping measurement}

The sample $\left(\mathrm{m}_{1} \mathrm{~g}\right)$ was weighed and poured into a measuring cylinder $\left(\mathrm{m}_{0} \mathrm{~g}\right)$ until it was approximately $8 / 10$ of the volume of the measuring cylinder, and then left at room temperature for 24 hours. The measuring cylinder was rotated $135^{\circ} \mathrm{C}$ from the upright position, poured for $60 \mathrm{~s}$, then inverted for $60 \mathrm{~s}$, and the mass $\left(\mathrm{m}_{2} \mathrm{~g}\right)$ of the measuring cylinder was weighed. The water was poured into the measuring cylinder until the volume of the measuring cylinder was $4 / 5$, covering the plug, and the measuring cylinder was turned upside down 10 times, then the contents were poured according to the above operation and the mass $\left(\mathrm{m}_{3} \mathrm{~g}\right)$ of the measuring cylinder was weighed. The residue $\mathrm{w}_{1}(\%)$ after pouring and the residue $\mathrm{w}_{2}(\%)$ after washing were calculated by formula (2) and formula (3).

$$
\begin{aligned}
& \mathrm{w}_{1}=\left(\mathrm{m}_{2}-\mathrm{m}_{1}\right) / \mathrm{m}_{0} \\
& \mathrm{w}_{2}=\left(\mathrm{m}_{3}-\mathrm{m}_{0}\right) / \mathrm{m}_{1}
\end{aligned}
$$

Where $\mathrm{m}_{1}$ was the mass of the sample; $\mathrm{m}_{2}$ was the total mass of the graduated cylinder, the grind plug and the residue after pouring; $\mathrm{m}_{3}$ was the total mass of the graduated cylinder, grind plug and residue after washing; and $\mathrm{m}_{0}$ was the mass of the measuring cylinder and the grinder after achieving a constant weight.

\subsubsection{Storage stability}

In accordance with the method specified in GB/T 19136-2003, the samples were placed in a $50{ }^{\circ} \mathrm{C}$ or $4{ }^{\circ} \mathrm{C}$ environment for 4 weeks, and the appearance of the samples 
was observed to see if there was delamination.

\subsection{5 $\mathrm{pH}$ measurement}

After the $\mathrm{pH}$ meter was calibrated, the sample $(1.0 \mathrm{~g})$ was placed in a $100 \mathrm{~mL}$ beaker and $100 \mathrm{~mL}$ of redistilled water was added; the mixture was stirred vigorously for $1 \mathrm{~min}$ and measured by the $\mathrm{pH}$ meter. All measurements were performed in triplicate.

\subsection{In vitro release of LC}

To study in vitro release of the LC from the microcapsules, $0.5000 \mathrm{~g}$ of LC-PDA CS and commercial CS were loaded in the dialysis bag respectively. They were placed into a wild-mouth flask with $95 \mathrm{ml}$ of ethanol/water (1:1). After then, the flask was placed in shaking incubator (SHZ-82 Digital display water bath thermostat, Jintan Kexing Instrument Factory, China) with the speed of $150 \mathrm{rpm} .3 \mathrm{~mL}$ of solvent outside the dialysis bag was collected over a period of $150 \mathrm{~h}$ at various time intervals and was replaced with $3 \mathrm{ml}$ fresh medium solution. The LC concentration was measured by UV-vis spectrophotometer at a wavelength of $278 \mathrm{~nm}$ to determine the release profile of $\mathrm{LC}$.

\section{The performance results of different microcapsule suspensions}

First, samples CS-1 and CS-13 showed a small amount of delamination after two weeks; samples CS-5 and CS-9 showed delamination after three weeks. It could be seen that when the content of the thickener was $0.1 \%$, the sample had poor room temperature stability. Theoretically, the higher the content of thickener, the less likely the system was to undergo delamination but also the less fluid the system was. The 
results of the orthogonal test were analyzed intuitively to examine the impact of various additives and their dosages on the suspensions. The results of the intuitionistic analysis are shown in Table S2.

As shown in Table S2, the effects of different factors on different indicators were different. Therefore, it was not feasible to unify the primary and secondary orders of the importance of the three factors on the three indicators. Although the optimal schemes corresponding with different indicators were different, the optimal scheme could be achieved through a comprehensive balance method. According to the results of the suspension rate, dumping performance and storage stability, the optimal scheme was CS-7, namely, the optimal formulation of $2.5 \%$ LC-PDA CS was that the proportion of LC-PDA MC was 5.0\%, antifreeze (ethylene glycol) content was 4\%, antifoaming agent (WACKER SRECN type) content was $0.2 \%$, preservative (sodium benzoate) content was $0.1 \%$, dispersing agent (SP-SC3260) content was 5.0\%, thickener (xanthan gum) content was $0.3 \%$ and the remainder was deionized water. 
Table S1. Orthogonal test design that the quantity of thickener and dispersing agent.

\begin{tabular}{ccccc} 
Thickener (\%) & \multicolumn{4}{c}{ Dispersing agent (\%) } \\
& $\mathbf{2 . 5}$ & $\mathbf{5 . 0}$ & $\mathbf{7 . 5}$ & $\mathbf{1 0 . 0}$ \\
$\mathbf{0 . 1}$ & CS-1 & CS-5 & CS-9 & CS-13 \\
$\mathbf{0 . 2}$ & CS-2 & CS-6 & CS-10 & CS-14 \\
$\mathbf{0 . 3}$ & CS-3 & CS-7 & CS-11 & CS-15 \\
$\mathbf{0 . 4}$ & CS-4 & CS-8 & CS-12 & $-a$ \\
\hline
\end{tabular}

${ }^{a}$ The total mass of the final formulation exceeds $100 \%$ if thickeners and dispersing agent were added in proportion of 'cs-16'.

Table S2. The performance results of different microcapsule suspensions.

\begin{tabular}{cccccc} 
No. & $\begin{array}{c}\text { Susp. rate } \\
(\%)\end{array}$ & $\begin{array}{c}\text { Pour resi. } \mathrm{W}_{1} \\
(\%)\end{array}$ & $\begin{array}{c}\text { Washing Resi. } \mathrm{W}_{2} \\
(\%)\end{array}$ & \multicolumn{2}{c}{ Storage stability } \\
& $\left(\% 0^{\circ} \mathrm{C}\right.$ & $4{ }^{\circ} \mathrm{C}$ \\
$\mathrm{CS}^{2}{ }^{b}$ & 37.3 & 6.3 & 1.4 & $\mathrm{~L}$ & $\mathrm{~N}$ \\
$\mathrm{CS}-3$ & 38.2 & 11.8 & 1.9 & $\mathrm{~N}$ & $\mathrm{~N}$ \\
$\mathrm{CS}-4$ & 17.8 & 26.8 & 7.2 & $\mathrm{~N}$ & $\mathrm{~N}$ \\
$\mathrm{CS}-6$ & 79.2 & 7.2 & 1.5 & $\mathrm{~L}$ & $\mathrm{~N}$ \\
$\mathrm{CS}-7$ & 84.0 & 10.0 & 3.0 & $\mathrm{~N}$ & $\mathrm{~N}$ \\
$\mathrm{CS}-8$ & 88.7 & 26.4 & 5.8 & $\mathrm{~N}$ & $\mathrm{~N}$ \\
$\mathrm{CS}-10$ & 61.6 & 7.5 & 1.8 & $\mathrm{~L}$ & $\mathrm{~N}$ \\
$\mathrm{CS}-11$ & 37.7 & 11.3 & 2.6 & $\mathrm{~N}$ & $\mathrm{~N}$ \\
$\mathrm{CS}-12$ & 16.5 & 19.5 & 6.2 & $\mathrm{~N}$ & $\mathrm{~N}$ \\
$\mathrm{CS}-14$ & 24.0 & 10.0 & 2.9 & $\mathrm{~N}$ & $\mathrm{~N}$ \\
$\mathrm{CS}-15$ & 35.2 & 7.2 & 2.3 & $\mathrm{~N}$ & $\mathrm{~N}$
\end{tabular}

${ }^{b}$ CS-1; CS-5; CS-9; CS-13 stratify at room temperature.

L: Layered N: Not-layered.

Table S3. Rheological fitting parameters of LC-PDA CS and commercial CS by Herschel-Bulkley model.

\begin{tabular}{ccccc}
\multicolumn{5}{c}{ Herschel - Bulkley Model } \\
Parameters & $\tau_{0}$ & $\mathrm{k}$ & $\mathrm{R}^{2}$ & $\mathrm{c}$ \\
LC-PDA CS & 0.429 & 1.614 & 0.985 & 0.337 \\
Commercial CS & 0.332 & 2.552 & 0.989 & 0.308
\end{tabular}

Table S4. The structural recovery rates of LC-PDA CS and commercial CS under the change of shear stress.

\begin{tabular}{cccccc}
\multicolumn{7}{c}{ Structural recovery rate (\%) } \\
Time (s) & 5 & 10 & 15 & 20 & 25 \\
LC-PDA CS & 87.99 & 90.26 & 91.10 & 91.56 & 91.99 \\
Commercial CS & 70.08 & 73.79 & 76.48 & 79.18 & 81.37
\end{tabular}


Table S5. Results of LC-PDA CS, LC and commercial CS against Lipaphis erysimi.

$\begin{array}{ccccc}\text { Sample } & \begin{array}{c}\text { Toxicity regression } \\ \text { equation }\end{array} & \begin{array}{c}\text { Correlation } \\ \text { coefficient }\end{array} & \mathrm{LC}_{50}{ }^{c}(\mathrm{ppm}) & 95 \% \text { Confidence limit }(\mathrm{ppm}) \\ \text { LC-PDA CS } & \mathrm{y}=5.8740+1.5598 \mathrm{x} & 0.9260 & 2.752 \times 10^{-1} & 1.126 \times 10^{-1}-6.724 \times 10^{-1} \\ \text { LC } & \mathrm{y}=7.9279+2.5413 \mathrm{x} & 0.9788 & 7.043 \times 10^{-2} & 4.764 \times 10^{-2}-1.042 \times 10^{-1} \\ \text { Commercial CS } & \mathrm{y}=2.6480+3.6053 \mathrm{x} & 0.8776 & 4.491 & 1.464-13.77\end{array}$

${ }^{c}$ Lethal concentration that causes $50 \%$ mortality

Table S6. Acute toxicity to aquatic organism of LC-PDA CS, commercial CS and LC.

\begin{tabular}{ccccc}
\multicolumn{1}{c}{ Sample } & $\begin{array}{c}\text { Toxicity regression } \\
\text { equation }\end{array}$ & $\begin{array}{c}\text { Correlation } \\
\text { coefficient }\end{array}$ & $\mathrm{LC}_{50}{ }^{c}(\mathrm{ppm})$ & $\begin{array}{c}95 \% \text { Confidence limit } \\
(\mathrm{ppm})\end{array}$ \\
LC-PDA CS & $\mathrm{y}=9.8825+2.2950 \mathrm{x}$ & 0.9778 & $7.456 \times 10^{-3}$ & $6.786 \times 10^{-3}-8.192 \times 10^{-3}$ \\
LC & $\mathrm{y}=13.0493+3.1114 \mathrm{x}$ & 0.9764 & $2.588 \times 10^{-3}$ & $2.244 \times 10^{-3}-2.984 \times 10^{-3}$ \\
Commercial CS & $\mathrm{y}=10.4293+5.4398 \mathrm{x}$ & 0.9823 & $1.004 \times 10^{-1}$ & $9.255 \times 10^{-2}-1.090 \times 10^{-1}$ \\
& & & &
\end{tabular}

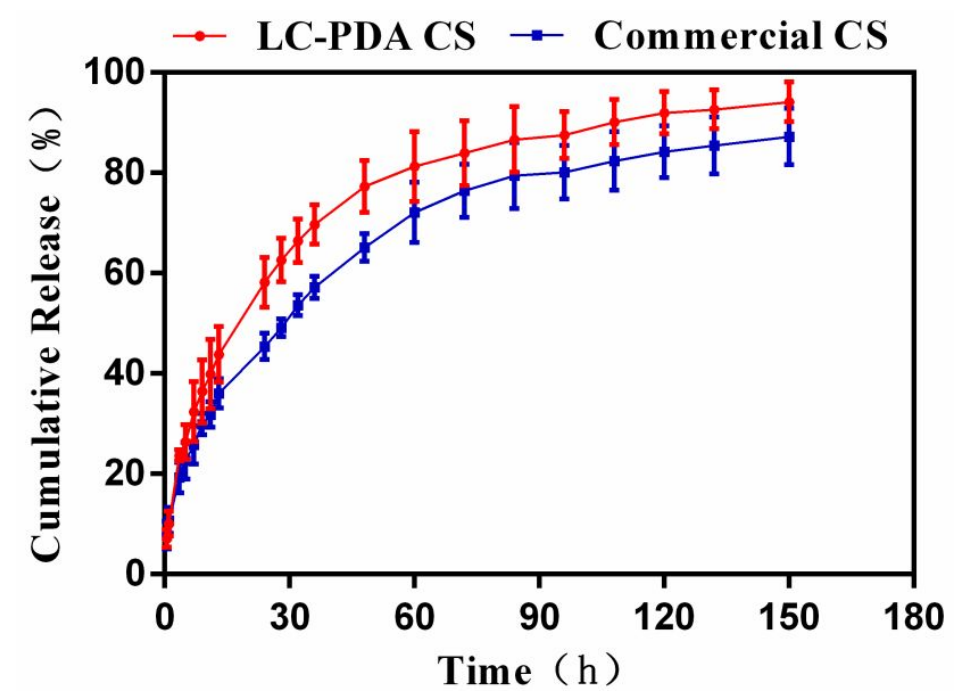

Figure S1. Release curves of LC from LC-PDA CS and commercial CS at the ethanol-water $(1: 1)$ medium.

\section{References}

(1) Zou, A.; Yang, Y.; Cheng, J.; Garamus, V. M.; Li, N., Construction and characterization of a novel sustained-Release delivery system for hydrophobic pesticides using biodegradable polydopamine-based micromicrocapsules. J. Agric. Food Chem. 2018, 66, 6262-6268.

(2) GB/T 14825-2006, Determination method of suspensibility for pesticides. 